\title{
Local application of biphosphonate as a biochemical barrier against bone graft resorption in mandibular reconstruction
}

\author{
Romzi Hanif*, Rahardjo**, Muhammad Masykur Rahmat**, Cahya Yustisia Hasan**® \\ ${ }^{*}$ RSUD Prof. Dr. Margono Soekarjo, Central Java, Indonesia \\ Oral and Maxillofacial Surgery Specialty Program, Faculty of Dentistry, Universitas Gadjah Mada, Yogyakarta, Indonesia \\ **Department of Oral and Maxillofacial Surgery, Faculty of Dentistry, Universitas Gadjah Mada, Yogyakarta, Indonesia \\ **JI Denta No 1, Sekip Utara, Yogyakarta, Indonesia; $₫$ correspondence: yustisiahasan@ugm.ac.id
}

Submitted: 11 ${ }^{\text {st }}$ March 2019; Revised: 22 ${ }^{\text {nd }}$ May 2019; Accepted: $3^{\text {rd }}$ October 2019

\begin{abstract}
Mandibular reconstruction to restore functions and aesthetics after mandibular resection remains a major surgical challenge. This type of reconstruction using non-vascularized autogenous bone graft harvested from iliac bone has been popular given that this bone is numerous and donor site morbidity can be minimized, however, it is highly resorbable. Local application of bisphosphonate by immersing bone graft in biphosphonate manages to inhibit bone graft resorption prior to the formation of new bone to support the osteogenesis and osteointegration of bone grafts. This paper aimed at examining the positive results of iliac bone graft osteogenesis and osteointegration following local application of bisphosphonate in mandibular reconstruction. A 22-year-old female patient came with a complaint of painless right cheek mass that has swollen slowly since 2 years. Radiographic examination showed cloudy radiolucent images in the right mandibular corpus to the right mandibular ramus, while histopathological examination indicated ossifying fibroma. Patient underwent mandibular resection followed by iliac bone graft-based mandibular reconstruction. Bone graft was immersed with bisphosphonate (zoledronate acid $0.005 \mathrm{mg} / \mathrm{ml}$ ) for 3 minutes, then rinsed with saline for 3 minutes, followed by fixing bone graft on the reconstruction plate. Postoperative follow up in the 36th week showed no signs of infection and dehiscence in the surgery site, and the radiographic examination indicated signs of osteogenesis and osteointegration of mandibular bone graft. Local application of biphosponate on bone graft promotes favorable results of osteogenesis and osteointegration in mandibular reconstruction.
\end{abstract}

Keywords: biphosponate; bone graft; mandibular reconstruction; osteogenesis; osteointegration

\section{INTRODUCTION}

Mandible is an essential anatomical structure for the physiological and aesthetic functions of humans. Therefore, any mandibular defects caused by tumors, malignancy, trauma, osteomyelitis, and osteoradionecrosis will considerably affect patient's quality of life. Hemimandibulectomy, a type of mandibular resection may result in mandibular defects leading to mandibular dysfunctions. ${ }^{1}$ The mandibular defects after hemimandibulectomy require reconstructions to restore mandibular aesthetics and functions. ${ }^{2}$

Mandibular reconstruction can be done by using reconstruction plate and iliac bone-derived non vascularized autogenous bone graft. ${ }^{3}$ The plate mostly used for mandibular reconstruction is a rigid plate that is applied along the inferior mandibular margin. ${ }^{4}$ Among the advantages of non-vascularized autogenous bone graft involving iliac bone include the availability in large quantities $\left(50-90 \mathrm{~cm}^{3}\right)$, shorter surgical and recovery period, no need for microvascular surgery skills, simultaneous surgery by 2 teams, minimal donor site morbidity, minimal patient's scar, various forms of harvest (block or particulate, cortical and corticocancellous), while the disadvantages are weak osteogenesis and excessive resorption in defects over $6 \mathrm{~cm}$ caused by the origin of endochondral and cortico-cancellous morphology. ${ }^{5,6}$

Biphosponate is a preparation for minimizing osteoclast activity when in contact with bone surfaces, especially in subjects with active resorption where 
biphosponate works as a biochemical barrier for bone resorption. Biphosponate may also inhibit resorption in bone graft. Studies involving experimental animals with biphosponate-locally applied bone graft showed the ability to protect graft from resorption without affecting other skeletal bones. ${ }^{7}$

Local application of biphosponate to bone graft succeeds in inhibiting excessive bone graft resorption and fostering new bone formation (osteogenesis). This article reported clinical and radiological evaluations of bone graft osteogenesis after local application of biphosponate to hemimandibulectomypost mandibular reconstruction.

\section{METHODS}

A 23-year-old female patient came to the oral surgery department of Dr. Sardjito General Hospital to check her right cheek swelling and numbness in her right lip. Clinical examination showed a facial asymmetry; there was swelling in the mandibular ramus area which was hard in consistency, and there was an area with an increase in excitatory threshold on the cheek of the inferior mandibular margin and paresthesia in the right lower lip The intraoral examination indicated an swelling in the rough-surfaced lingual bone of region 44 to 48 with the same gingival color as the surrounding tissue Figure 1.

Panoramic radiograph showed the presence of cloud of smoke in radiolucent lesions in the mandibular corpus region until the right mandibular ramus along with bone expansion and uneven angular inferior margin and resorption of tooth 46 and 47 roots. Examination of 3D MSCT showed lesions in the mandibular ramus to the right mandibular subcondyle and bone destruction occurred in the corpus lingual side to the right mandibular ramus Figure 2. The examination of anatomic pathology showed trabecular-arranged bone tissue with osteoblastic rimming, connective tissue stroma with proliferation of fibroblasts, which was declared as ossifying fibroma.

This case was diagnosed as right mandibular ossifying fibroma. Right mandibular hemimandibulectomy and mandibular reconstruction were chosen as the treatment involving reconstruction plate and non-vascularized autogenous bone graft derived from the iliac bone along with local application of biphosponate on the bone graft. The surgery was carried out in collaboration with the orthopedic department to harvest a $3 \mathrm{~cm} \times 5 \mathrm{~cm} \times 1.5 \mathrm{~cm}$ iliac graft.

Hemimandibulectomy was performed by cutting the mandible from the tooth region 44, followed by mandibular reconstruction with mounted reconstruction plate. Harvested iliac graft was soaked in biphosponate preparation, zoledronate $0.005 \mathrm{mg} / \mathrm{ml}$ for 3 minutes, after which it was rinsed with saline. The graft was then fitted to the reconstruction plate with the cortical side facing the reconstruction plate Figure 3 . The patient underwent inpatient post-operative treatment for 4 days and intermaxillary wiring was fixed and retained for 6 weeks.

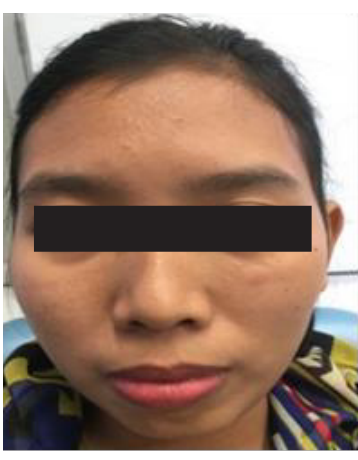

(A)

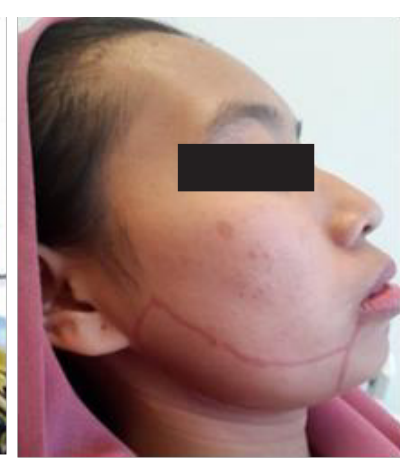

(B)

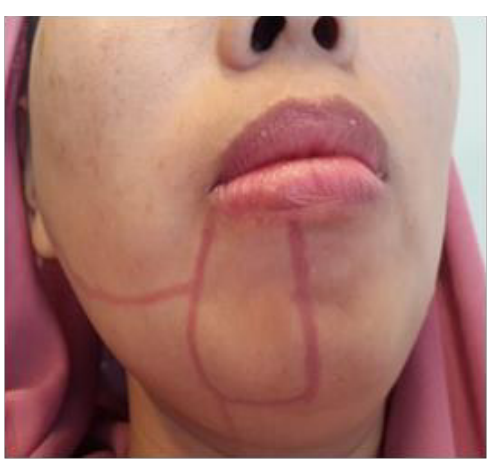

(C)

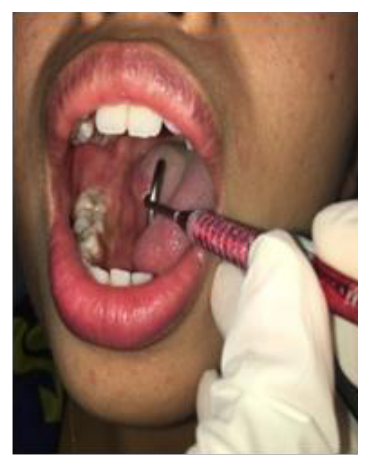

(D)

Figure 1. (A) facial asymmetry, (B) area with an increased excitatory threshold, (C) area of paresthesia, (D) swelling of uneven-surfaced lingual bone of region 44 to 48 

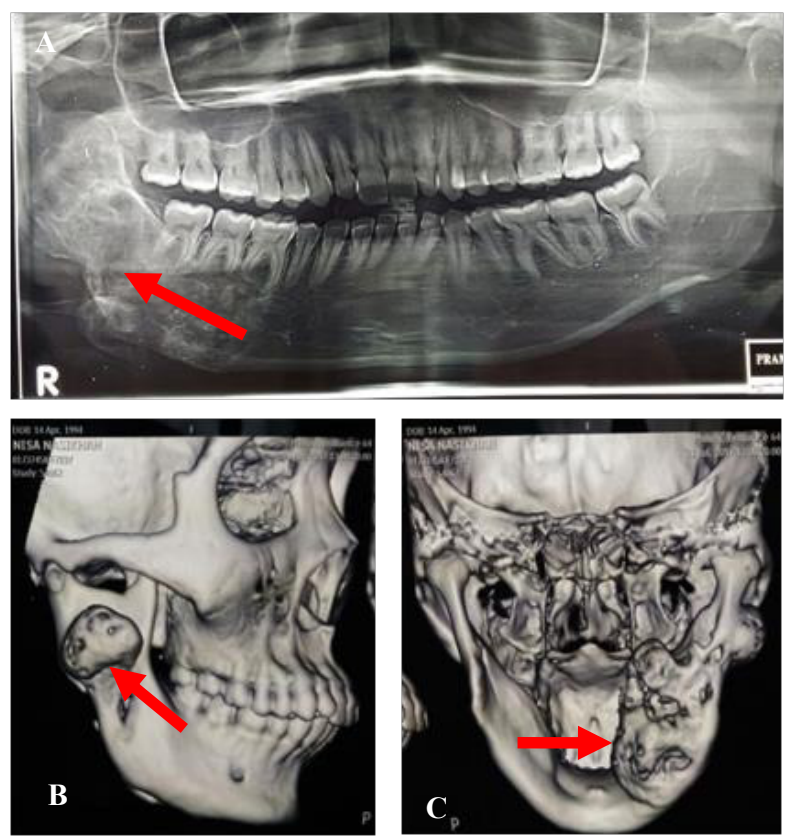

Figure 2. (A) Diffuse cloud of smoke lesions on the ascending corpus-ramus in right mandibular angles (red arrow), (B) and (C) lesions of bone destruction in the lingual side of the corpus to the right ascending in the right mandible (red arrow).
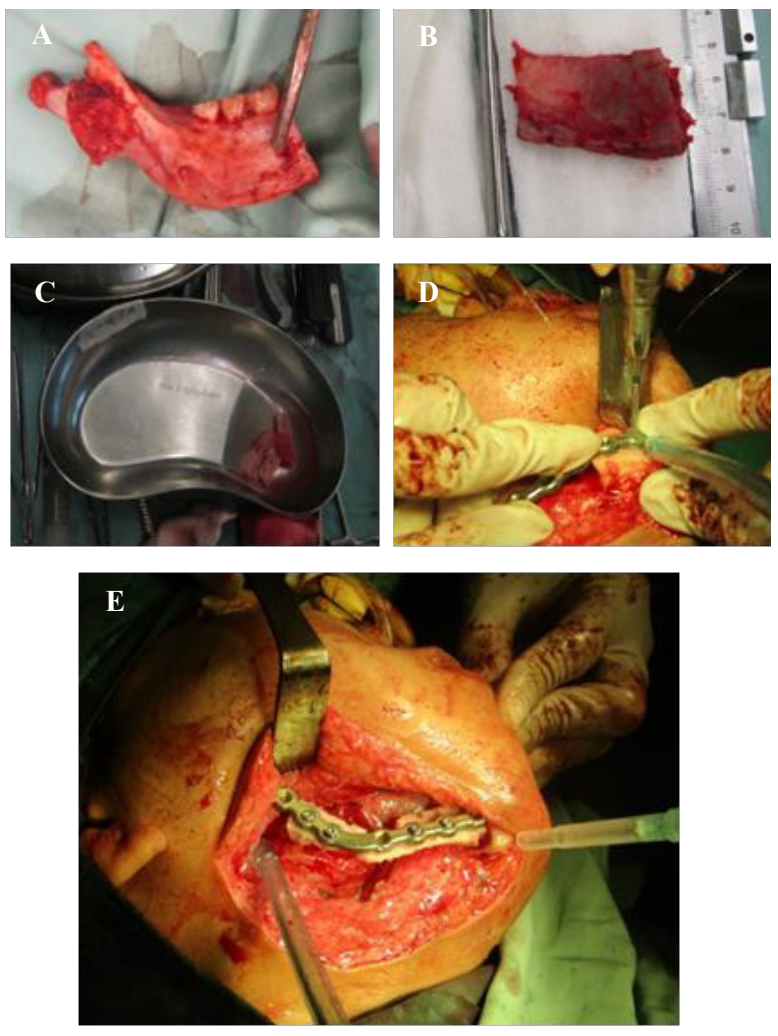

Figure 3. (A) hemimandibulectomy-post mandibular segment, (B) bicortical iliac bone graft, (C) iliac graft soaked in Zometa for 3 minutes, subsequently rinsed with $0.9 \% \mathrm{NaCl}$, (D) mounted bridge plate on healthy bone with a 3 -hole $2.4 \times 10 \mathrm{~mm}$ screw, (E) fitting iliac graft into the reconstruction plate.
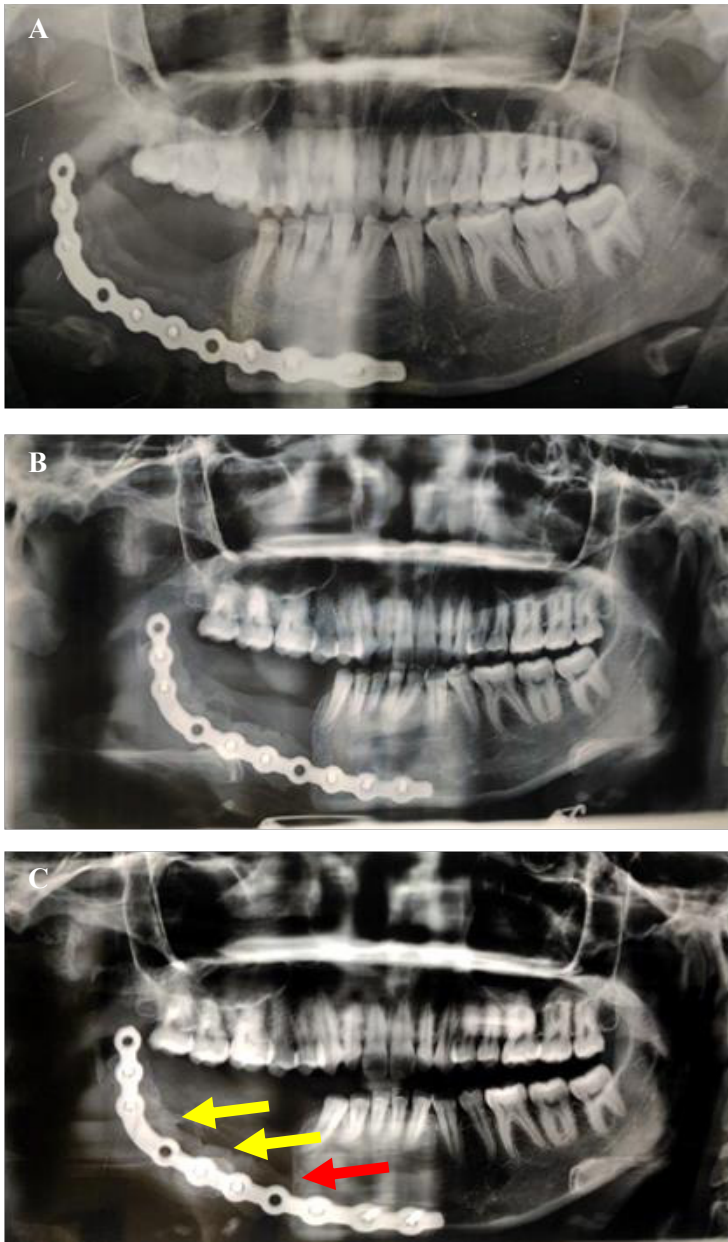

Figure 4. (A) panoramic x-ray of mandibular reconstruction taken in week 12 post-opeative, (B) panoramic x-ray of mandibular reconstruction taken in week 24 post-opeative, (C) panoramic x-ray of mandibular reconstruction taken in week 36 post-opeative showed osteointegration in the mandibular bone with graft (red arrow) and signs of osteogenesis along the graft (yellow arrow)

Clinical evaluation in week 6 following the removal of the intermaxillary wiring showed neither dehiscence nor signs of intraoral and extraoral infection and left side occlusion was well formed. Evaluation of the panoramic radiographs taken in week 12 and 24 postoperative showed that a reconstruction plate was fitted to the right mandible and a graft image along the reconstruction plate with minimal graft resorption was seen. In week 36 postoperative, the panoramic radiograph was evaluated, resulting in signs of osteointegration in the mandibular bone with graft in the distal area of tooth 43 , and signs of osteogenesis along the graft attached to the reconstruction plate Figure 4 . 
Majalah Kedokteran Gigi Indonesia. December 2019; 5(3): 143 - 148

ISSN 2460-0164 (print)

ISSN 2442-2576 (online)

\section{DISCUSSION}

Hemimandibulectomy is highly likely to result in mandibular defects. Mandibular reconstruction to restore the patient's anatomical function and shape must be done immediately after resection..$^{8,9}$ Hemimandibulectomy, the radical resection along with reconstruction was opted for this case by considering lesions with the involvement of the lingual and buccal sides from the mandibular corpus to the right mandibular ascending ramus.

Ossifying fibroma is a benign tumor that belongs to a type of fibro-osseous lesion. Women, in their twenties, are more prone to this fibroma than men. Ossifying fibroma is most commonly found in the mandible especially in the posterior area or molar region $10 .{ }^{10}$

Ossifying fibroma is slow but persistent in nature and causes expansion and the thinning of the buccal and lingual cortical layers to the lower mandibular inferior margin. This fibroma triggers no pain but paresthesia when occurring in the nerve structures. The oral epithelium will remain intact unless there is a secondary infection. ${ }^{10-12}$

Lesion of ossifying fibroma must be completely excised from the surrounding bone because of the high recurrence risk and proneness to malignancy. ${ }^{13}$ The possible method for treating ossifying fibroma is surgical excision for small-sized lesions with clear boundaries, whereas large ones require radical surgery by involving the boundaries of surrounding healthy tissue and reconstruction. ${ }^{11,14}$ Hemimandibulectomy-based radical resection along with reconstruction was chosen to fit the condition of bone destruction and recurrence risk.

The opted method of mandibular reconstruction was a titanium plate, with non-vascularized autogenous bone graft derived from the iliac bone. Mandibular reconstruction plates and screw are the most widely used alloplastic materials for the reconstruction of the mandibular defects. The method is an easy, reliable procedure, and can be used for subsequent rehabilitation. ${ }^{15,16}$

Iliac bone serves as a graft donor for this case because it is numerous and relatively low in morbidity. Given that iliac bone is far from the facial region, it is possible for two teams to operate simultaneously for efficient surgery. ${ }^{17}$

Prior to implantation, iliac bone graft was measured and shaped to fit into the reconstruction plate. The iliac graft was immersed in zometa solution $0.005 \mathrm{mg} / \mathrm{ml}$ for 3 minutes, after which it was rinsed with normal saline. Zometa is a trademark for biphosponate preparation which contains zoledronate, a part of amino biphosponate, with nitrogen in the atomic chain of atoms and molecule. The local application of zoledronatederived biphosponate is expected to inhibit the excessive bone graft recession which occur before osteogenesis during bone graft. Local application of zelodronate $0.005 \mathrm{mg} / \mathrm{ml}$ in bone graft was done by immersing the graft for 3 minutes, followed by rinsing for 3 minutes in the experimental animal and resulted in slow resorption in the bone graft and an increase in new bone formation around the graft. ${ }^{18}$

Nitrogen-containing biphosponates such as pamidronate, olpadronate, ibandronate, alendronate, risedronate and zoledronate have a working mechanism that inhibits farnesyl diphosphate synthesis which causes failure of prenylation (fatty acid chain transfer) of various intracellular proteins, especially small GTP binding protein (Trimeric G-protein). Prenylation failure will trigger a premature apoptotic process of osteoclast cells resulting in inability for bone resorption. ${ }^{18,19}$

Experimental studies showed that aplication of biphosponate can be performed by systemic administration of biphosponate or local aplication of biphosponate. One of the problems associated with systemic administration of biphosponate is the development of microcracks, which might be caused by excessive mineralization. Another problem associated with systemic bisphosphonate treatment is osteonecrosis of the jaw. It was suggested that the incidence of osteonecrosis of the jaw is higher in zoledronic acid treatment than in treatment using other bisphosphonates. Systemic administration of bisphosphonates only reach vascularized bone and the necrotic, non-vascularized bonegraft can only be reached by the bisphosphonate once it has been revascularised. Furthermore, blocking necrotic bone resorption, as in the case of a bone graft, may 
require higher local concentrations, which may be difficult to achieve with systemic treatment. Such problems would be solved if the graft could be treated by local aplication of biphosponate before implantation. ${ }^{20,21}$

Experimental studies of bone graft locally applied with biphosponate showed the ability to protect graft from resorption without any effects on other skeletal bones. ${ }^{7}$ Locally applied biphosponate with zometa (zoledronate $0.005 \mathrm{mg} / \mathrm{ml}$ ) for 3 minutes in experimental dogs resulted in an increase in new bone formation in the group applied with zometa compared to the control group. ${ }^{7,18,22}$ Local application of biphosponate to alveolar bone defect is able to minimize alveolar bone resorption and improve new bone formation in the alveolar defect and foster the regeneration capacity of biomaterials and bone density.

Topical application of amino-biphosponate solution to bone defects or post-extraction socket, either alone or combined with a bone graft, is a risk-free procedure as biphosponate contributes to the initial phase of bone healing and is absorbed mainly by the attached bone so tiny amount may be released into circulation. ${ }^{22}$

Unfortunately, there are only few studies on local application of biphosponate in humans. In the previous study, it was done by immersing the allograft bone in $10 \mathrm{ml}$ Bonefos (disodium clodronate) in a dose of $60 \mathrm{mg} / \mathrm{ml}$ for 3 minutes then rinsed with $500 \mathrm{ml}$ normal saline in pelvic revision surgery. The $24^{\text {th }}$ month postoperative control showed that rinsing morselized bone graft soaked in biphosponate could prevent resorption and minimize the risk of mechanical failure, whereas in the control group, the graft experienced resorption after 3 months. ${ }^{7,20}$

Radiographic evaluation of post-reconstruction mandibular bone graft in the $36^{\text {th }}$ week postoperative showed minimal bone graft resorption and signs of osteogenesis along the surface of the bone graft and osteointegration occurred in the mandibular bone with graft in the distal area of tooth 43 . The clinical evaluation indicated that locally-applied biphosponate was proven to minimize complications in the soft tissue or hard tissue around the bone graft.

\section{CONCLUSION}

Local application of biphosponate by immersing iliac bone graft in biphosponate preparations used in mandibular reconstruction-post hemimandibulectomy is proven to inhibit premature bone graft resorption and increase osteogenesis along the bone graft surface and osteointegration of bone graft. The use of locally-applied biphosponate is proven to minimize complications in the soft tissue or hard tissue around bone graft.

\section{ACKNOWLEDGMENT}

Gratitude and appreciation are extended to all the faculty members of the Department of Oral and Maxillofacial Surgery, Faculty of Dentistry, Universitas Gadjah Mada, Indonesia for good cooperation in the medication therapy management for this case study.

\section{REFERENCES}

1. Hupp JR, Ellis E, Tucker MR. Contemporary oral and maxillofacial surgery 5th ed. St.Louis Missouri: Mosby Elsevier; 2008. 449-468.

2. Fernandes RP, Yetzer JG. Reconstruction of acquired oromandibular defect. Oral Maxillofac Surg Clin North Am. 2013; 25(2): 214-249. doi: 10.1016/j.coms.2013.02.003

3. Farwell DG, Futran ND. Oromandibular reconstruction. Facial Plast Surg. 2000; 16(2): 115-126. doi: 10.1055/s-2000-12573

4. Wong $\mathrm{RC}$, Tideman $\mathrm{H}$, Kin L, Merkx MA. Biomechanics of mandibular reconstruction: a review. IJOMS. 2009; 39(4): 313-319. doi: 10.1016/j.ijom.2009.11.003

5. Chim H, Salgado CJ, Mardini S, Chen HC. Reconstruction of mandibular defects. Semin Plast Surg. 2010; 24(2): 188-197. doi: 10.1055/s-0030-1255336

6. Mounir M, Abou-ElFetouh A, El-Beialy W, Faramawey M, Mounir R. Vascularized versus non-vascularized autogenous bone graft for immediate reconstruction of segmental mandibular defects: a systematic review. OHDM. 2015; 14(6): 337-349.

7. Kesteris $U$ and Aspenberg P. Rinsing morcellised bone grafts with biphosphonate 
solution prevents their resorption: a prospective randomized double-blinded study. J Bone Joint Surg Br. 2006; 88(8): 993-996. doi: 10.1302/0301-620X.88B8.17457

8. Alfotawi $\mathrm{R}$, Ayoub $\mathrm{A}$. Reconstruction of maxillofacial bone defects: contemporary methods and future techniques. Am J Adv In Med Sc. 2014; 2(1): 18-27.

9. Buchbinder D, Arken M. Mandibular reconstruction. Head \& Neck Surg-Otolaryngol. 1993; 2: 1980-1999.

10. Martín-Granizo R, Sánchez-Cuellar A, Falahat F. Cemento ossifying fibroma of the upper gingivae. Otolaryngol Head Neck Surg. 2000; 122(5): 775. doi: 10.1016/S0194-5998(00)70216-6

11. Triantafilidou K, Karakinaris G, Psomaderis K, Iordanisdis F, Karakasis D. Fibrous dysplasia of craniomaxillofacial bones a clinical study of 18 cases and review of the literature. BJDM. 2014; 18(2): 99-105.

doi: $0.1515 /$ bjdm-2015-0016

12. Gondivkar SM, Gadbail AR, Chole R, Parikh $\mathrm{RV}$, Balsaraf S. Ossifying fibroma of the jaws: report of two cases and literature review. Oral Oncol. 2011; 47(9): 804-809.

doi: 10.1016/j.oraloncology.2011.06.014

13. Toyosawa S, Yuki M, Kishino M, Ogawa $Y$, Ueda T, Murakami S, Konishi E, Lida S, Kogo M, Komori T, Tomita Y. Ossifying fibroma vs fibrous dysplasia of the jaw: molecular and immunological characterization. Mod Pathol. 2007; 20(3): 389-396.

doi: $10.1038 /$ modpathol.3800753

14. Chang $\mathrm{C}$, Hung $\mathrm{H}$, Chang J, Yu CH, Wang $\mathrm{Y}$, Liu B, Chiang C. Central ossifying fibroma: clinicopathologic study of 28 cases. J Formos Med Assoc. 2008; 107(4): 288-294.

doi: 10.1016/S0929-6646(08)60089-3

15. Del-Hoyo JA, Sanroman JF, Bueno PR, Gonzales FJD, Usandizaga JLGD, Gil FM, Gias LN, Lopez AC, Cardozo RM. Primary mandibular reconstruction with bridging plates. J Cranio-Maxillofac Surg. 1994; 22(1): 43-48. doi: 10.1016/S1010-5182(05)80295-1
16. Stosic S. Mandibular reconstruction state of the art and perspectives. Vojnosanit Pregel. 2008; 65(5): 397-403. doi: 10.2298/vsp0805397s

17. Elsalanty ME and Genecov DG. Bone graft in craniofacial surgery. Craniomaxillofac Trauma Recons. 2009; 2(3): 125-134. doi: 10.1055/s-0029-1215875

18. Hong C, Quach A, Lin L, Olson J, Kwon T, Bezouglaia O, Tran J, Hoang M, Bui K, Kim RH, Tetradis S. Local vs systemic administration of biphosphonates in rat cleft bone graft: a comparative study. PLoS One. 2018; 13(1): e0190901. doi: 10.1371/journal.pone.0190901

19. Shaw NJ, Bishop NJ. Bisphosphonate treatment of bone disease. Arch Dis Child. 2005; 90(5): 494-499. doi: 10.1136/adc.2003.036590

20. Mathijssen NM, Buma P, Hannink G. Combining bisphosphonates with allograft bone for implant fixation. Cell Tissue Bank. 2014; 15(3): 329-36. doi: 10.1007/s10561-013-9416-7

21. Jakobsen T, Baas J, Bechtold J. The effect of soaking allograft in bisphosphonate: a pilot dose-response study. Clin Orthop Relat Res. 2010; 468(3): 867-874. doi: 10.1007/s11999-009-1099-9

22. Lozano-Carrascal N, Salomo-Coll O, Hernandez-Alfaro F, Gehrke SA, GargalloAlbiol J, Calvo-Guirado JL. Do topical applications of biphosphonates improve bone formation in oral implantology? a systematic review. Med Oral Patol Oral Cir Bucal. 2017; 22(4): e512-e519. doi: $10.4317 /$ medoral.21887. 